The aim of the present study was to characterize the neurophysiological profile of cognitive impairment associated with patients with chronic alcoholic and nonalcoholic liver disease. The authors evaluated 43 patients with cirrhotic liver disease: 19 patients with chronic alcohol ingestion and 24 nonalcoholic patients who had been infected with hepatitis B or C virus. Eleven healthy subjects were included as control subjects. A battery of 12 psychological tests was used to investigate cognitive deficits in the patients with chronic liver disease. It was observed that alcoholic patients with chronic liver disease showed a more important cognitive deterioration than those affected by hepatitis B or C virus.

(The Journal of Neuropsychiatry and Clinical Neurosciences 2014; 26:241-248)

\section{Cognitive Function in Patients With Alcoholic and Nonalcoholic Chronic Liver Disease}

\author{
Carlos Brodersen, M.D. \\ Eduardo Koen, M.D. \\ Alicia Ponte, M.D. \\ Silvina Sánchez, M.D. \\ Eduardo Segal, M.D. \\ Alberto Chiapella, M.D. \\ Maria Fernández \\ Maria Torres, M.D. \\ Valeria Tripodi, Dr. in Biochemistry \\ Abraham Lemberg, M.D.
}

\begin{abstract}
7 he CNS is usually involved during the development 1 of chronic liver disease. Depending on its degree of severity, this involvement can cause limited mental capacity, changes in psychomotility, and/or hepatic coma associated with irreversible brain damage. ${ }^{1}$

The initial signs are reversible mental and neurological disturbances that are not initially clinically clearly manifested and can be detected only by psychometric tests. This condition is called minimal or subclinical hepatic encephalopathy. Neuropsychological studies in cirrhotic patients with minimal hepatic encephalopathy have exposed a characteristic pattern of cognitive dysfunction. ${ }^{2-8}$

Prolonged ingestion of alcoholic beverages is known to cause damage in several tissues, altering their morphology and function. The liver, where alcohol metabolism takes place, is the principal target of alcohol toxicity, and the CNS is also implicated in alcohol damage. ${ }^{9,10}$
\end{abstract}

Received April 13, 2012; revised March 25, April 8, and May 2, 2013; accepted May 3, 2013. From the Gastroenterology Division, Hospital Durand, Buenos Aires, Argentina (CB); the Psychology Service, Hospital Durand, Buenos Aires, Argentina (EK, AP, E Sánchez, E Segal, AC); the Dept. of Pathophysiology, School of Pharmacy and Biochemistry, University of Buenos Aires, Buenos Aires, Argentina (MF, MT, AL); the Dept. of Analytical Chemistry, School of Pharmacy and Biochemistry, University of Buenos Aires, Buenos Aires, Argentina (VT); and the Consejo Nacional de Investigaciones Científicas y Tecnológicas, CONICET, Buenos Aires, Argentina (VT). Send correspondence to Abraham Lemberg, M.D.; e-mail: alemberg@ffyb.uba.ar

Copyright (c) 2014 American Psychiatric Association 
Acetaldehyde, the main metabolite of ethanol, has been shown to be able to bind covalently to liver proteins and may be responsible for the diverse toxic effects of ethanol. Acetaldehyde reacts with proteins via the $\varepsilon$-amino group of a lysine residue to yield hybrid malondialdehydeacetaldehyde-protein malondialdehyde and 4-hydroxy-2nonenal alcohol metabolites. These substances induce severe metabolic alterations and cause oxidative damage, including mitochondrial DNA damage, through the production of reactive oxygen species, in almost all mammalian tissue.

The presence of chronic viral toxins such as those of hepatitis B and C virus may also affect several extrahepatic tissues, including the CNS. ${ }^{11}$

In their evolution, both etiologies (i.e., the alcoholic one and the viral one) often cause severe complications, such as the development of portal vein hypertension in the splanchnic area, and consequently, the presence of a hyperdynamic systemic circulation, as well as neuropsychiatric dysfunction.

Tarter et al. ${ }^{12}$ considered that hepatic encephalopathy is a neuropsychiatric illness coexisting with morphological alterations and metabolic dysfunction that does not present full recovery after liver transplantation.

Pantiga et al. ${ }^{13}$ studied cognitive deficits associated with hepatic cirrhosis by applying neuropsychological tests to assess mental deficits in patients with different stages of hepatic cirrhosis and in liver transplant recipients. These authors showed that the severity of the cognitive impairment is linked to the amount of hepatic dysfunction. Liver transplant recipients showed only some degree of cerebral dysfunction in comparison to the control group.

Glucose is fundamental to develop a normal function of the brain. Basically, glucose comes in adequate amounts from liver glycogen hydrolysis. The cirrhotic liver is characterized by the destruction of an important quantity of hepatocytes, where glycogen is synthesized and stored, causing a deficient nutritional condition. It is probable that, in this deficient nutritional condition, other sources of glucose (e.g., muscles) are not satisfactory enough to cover glucose needs.

Conversely, the presence of cirrhotic livers in both etiologies suggests that it is probable that glycogenolysis is diminished due to parenchymal destruction. Then, the fundamental presence and use of glucose is decreased in neurons and astrocytes. Cyclic GMP and cAMP are substances that participate in glycogenolysis and maintain blood and most tissue glucose levels. In liver cirrhosis, the glutamine-glutamate cycle is also altered, and cyclic GMP and cAMP cannot produce their hyperglycemic activity. ${ }^{14}$
Presumably, these alterations in the normal metabolism of glucose could also generate cognitive disorders.

Numerous authors have searched for an efficient battery of psychological tests to distinguish mild cognitive impairment. A desired battery of psychological tests should combine convenience, practicability, sensitivity, specificity, low cost, and a high probability of standardization.

In this study, the neuropsychological profile of cognitive impairment associated with chronic liver disease was characterized by applying suitable psychological tests with the purpose to evaluate the intellectual function in this type of patients.

The aim of this experiment was to recognize cognitive dysfunction present in chronic liver disease, produced by prolonged alcohol ingestion or by hepatitis B and C virus infections, from the results obtained in the tests applied to patients with either etiology.

\section{METHODS}

\section{Patients}

Forty-three patients, with clinical and biochemical assessment of compensated cirrhosis (no bleeding, no ascites, and no other serious complications) were included in this study. Forty patients had histological evidence, whereas in three patients, the coagulation parameters could not be confirmed anatomically by liver biopsy. In 19 of these 43 patients, the disease was the consequence of chronic alcohol ingestion (more than 12 years of alcohol dependence), whereas in 24 patients, it was the consequence of chronic evolution of hepatitis B virus and hepatitis $C$ virus (from 6 months to 1 year for the appearance of clinical symptoms). To participate in this experiment, the patients were personally informed about the study and signed their consent before being included in this study. This project was submitted to the Committee of Ethics and Investigation of the hospital and accepted.

Patients and controls were submitted to usual laboratory tests, X-ray, ultrasonography, and other noninvasive examinations to confirm the patient's chronic disease. Immunochemical tests for viral infection or immune disease were used to verify the disease etiology. None of the patients or control subjects received parenteral nutrition or neuro-active medication in the 30 days before the study.

All the patients were submitted to a battery of 12 psychometric and verbal tests, and their performance was compared with that of 11 control subjects with no liver disease or mental pathology. 
Our patients with chronic liver disease presented different and important degrees of liver cirrhosis. All of them had portal hypertension, and some of them had bleeding esophageal varices during evolution. Chronic alcoholic or viral-infected patients belonged to the CHILD A (15 patients) and CHILD B (6 patients) classifications. CHILD classification is used to assess the prognosis of chronic liver disease using biochemical and clinical parameters such as total bilirubin, albumin, prothrombine time, ascites, and hepatic encephalopathy. Although it was originally used to predict mortality during surgery, it is now used to determine the prognosis, as well as the required strength of treatment and the necessity of liver transplantation. Chronic liver disease is classified into CHILD classes A-C (better to worse prognosis) using scores that have been previously defined. ${ }^{15}$

All the patients were selected from the out-patients of the Durand Hospital of Buenos Aires City (Argentina) and were divided into three groups: group A, 19 patients $(37.5 \%)$ with chronic alcoholic ingestion and liver disease (the patients were still consuming alcohol at the moment of their inclusion in our study); group B, 24 patients $(42.9 \%)$ with chronic liver disease after hepatitis $B$ and $C$ virus infection; group C, 11 controls with no liver disease or mental pathology and mild general complaints (this group had no pathological alcohol consumption).

Twenty-nine (53.7\%) of the subjects studied were women, whereas $25(46.3 \%)$ were men. Female age averaged 33.7 years $(\mathrm{SD}=12.1)$, whereas male age averaged 55.7 years $(\mathrm{SD}=11.5)$.

The patients included in this study had similar nutritional status, education, and socioeconomic situation.

\section{PSYCHOLOGICAL TESTS}

We designed a battery of neuropsychological tests that included memory tests (long-time memory test) and the following widely used measures: autoanalysis of episodes obtained from the patient's life, the Mini-Mental State Examination (MMSE), ${ }^{16}$ The Reitan's Number Connection Test, ${ }^{17,18}$ the Boston Naming Test, ${ }^{19}$ the Wechsler Adult Intelligence Scale (WAIS), including subtypes (number repetition, vocabulary, conceptual ability in similarities, and constructional capacity), the WAIS-Perceptual speed subtest of conceptual similarity ability, the Wechler Memory Scale, figural visual scanning, the Buschke selective task, learning capacity (consistent long-term retrieval test), evocation (long-term retrieval test), and word fluency (FAS test). All patients were investigated by the same experienced neuropsychologist (blind experiment) in a quiet room under adequate light and comfortable conditions. Each patient and control subject were interviewed three times.

Autoanalysis of Episodes Obtained From the Patient's Life MMSE or Folstein Test This test is a brief 30-point questionnaire used to screen for cognitive impairment and dementia or estimate the severity of cognitive impairment at a given point in time and to follow the course of cognitive changes in an individual over time. The MMSE test includes simple questions and problems in a number of areas: the time and place of the test, repeating lists of words, arithmetic such as the serial sevens, language use and comprehension, and basic motor skills.

The Reitan's Number Connection Test This test derives from the trail making test ${ }^{18}$ and measures cognitive motor abilities. In the number connection test, subjects have to connect numbers printed on paper consecutively from 1 to 25 , as quickly as possible. Errors are not enumerated, but patients are instructed to return to the preceding correct number and then carry on. The test score is the time the patient needs to perform the test, including the time needed to correct the errors. A low score indicates a good performance.

The Boston Naming Test This is the most widely used test of visual confrontation naming. The standard 60-item version has been used to assess language performance in participants with aphasia or dementia. Deficits in naming performance frequently appear in the first stages of Alzheimer's disease and increase with time. For this reason, the Boston Naming Test is one of the tests most frequently used to detect the disease and follow its evolution.

\section{Statistical Analysis}

Data were inserted in an Excel-type database and analyzed by a microprocessor Pentium III. Student's t test, analysis of variance (ANOVA), and Scheffe test were applied; $p<0.05$ was considered significant.

\section{RESULTS}

Groups A and B presented a low cognitive score compared with controls $(\mathrm{p}<0.05)$ (Figure 1$)$. 
FIGURE 1. WAIS-Perceptual Speed Subtest of Conceptual Similarity Ability

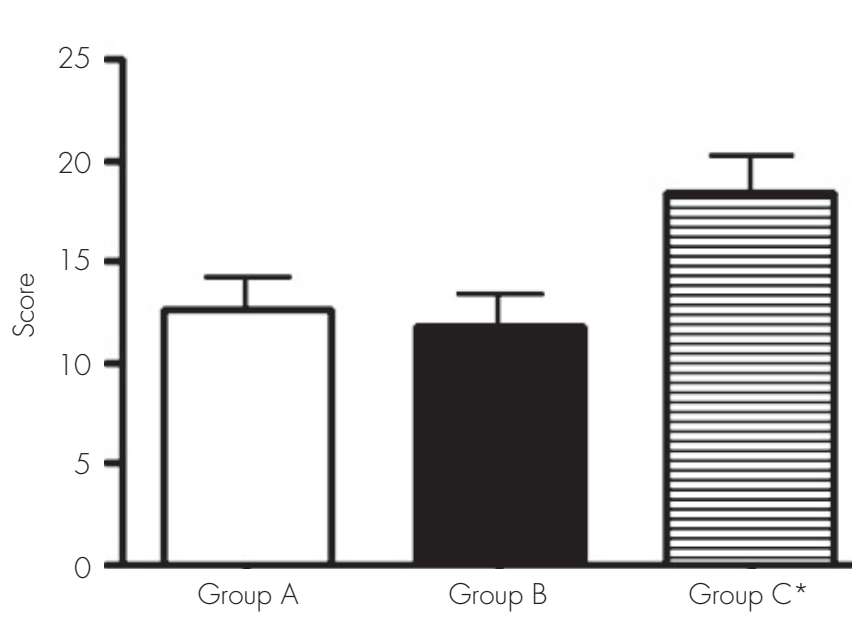

The main intellectual defects were recorded when the tests were related to praxis and velocity of mental processes $(\mathrm{p}<0.05)$ (Figures 2-4).

According to Figure 3 and applying the Reitan's test, a slower speed was seen in the response of patients with chronic hepatitis.

Our patients did not exhibit a significant failure in memory when their memory was tested for short and long periods of time or their visual memory was tested.

Cognitive dysfunction was documented when the techniques used involved spatial relationship with constructional ability, copy capacity, and velocity of mental processes. Our patients with chronic liver disease also showed an important disturbance in the visual motor area (coordination between visual, motor, and spatial behaviors).

Both groups with a chronic liver pathology showed clear impairment in the comprehensive capacity and the associative capacity in verbal concepts, indicating the presence of a deficit in conceptual judgment.

During the study of language (vocabulary) evaluation, we found a significant difference between patients and control subjects when submitted to the same test. As previously noted, all groups, including control subjects, had comparable sociocultural levels.

When the Boston Naming Test was applied to patients with chronic liver disease, we found that they required a longer time for evaluation, indicating the existence of a slowness of thoughts: the patients needed the presence of the object to recall the name of it (paraphasis). These patients seem to have impairment for the capacity to search information.
FIGURE 2. Figural Visual Scanning

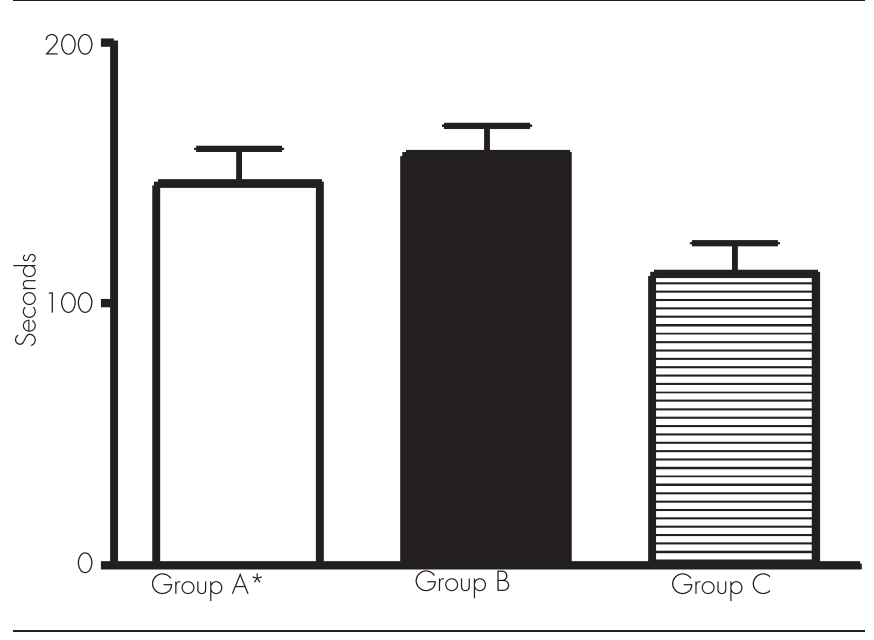

The application of these tests allowed us to find that patients with chronic liver disease present marked difficulties in attention and concentration.

\section{DISCUSSION}

The clinical presentation of hepatic encephalopathy in patients with chronic liver disease, especially in decompensated cirrhosis, includes alteration of consciousness, accompanied with loss of facial expression and speech disturbances together with a compromised motor system, represented by asterixis, tremor, increased tendon reflexes, increased muscle tone, and ataxic gait. In contrast, patients with minimal hepatic encephalopathy appear to have a normal mental state but subclinical cognitive alterations that could complicate their normal day-to-day tasks such as driving, operating equipment, and work-related activities.

Cognitive dysfunction is considered the failure of certain mechanisms such as understanding, coordination, spatial perception and reality, and problem solving. These limitations occur in the patient's personal, social, and work life to a greater or lesser degree. In turn, it is possible that the progression of cognitive impairment in life has emotional consequences for the patient, who, because of his/her limitations, may develop depressive symptoms, inhibitions, and/or impaired volition. All nerve cells are targets of alcohol and its metabolite agents and viral toxins, producing functional alterations. However, patients with a history of prolonged alcoholic intake showed more severe intellectual derangements than patients with chronic viral liver disease, except for 
FIGURE 3. Reitan's Test

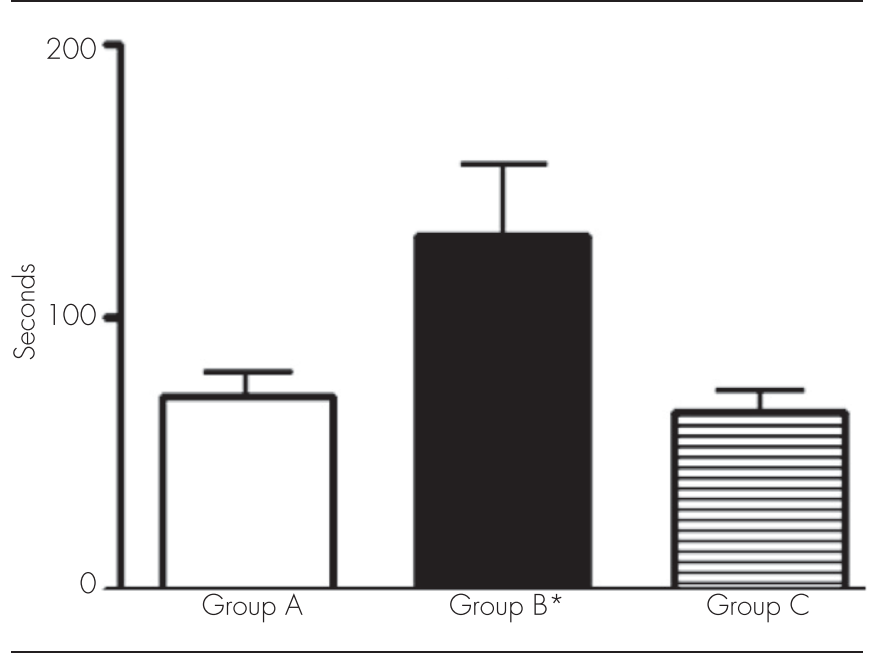

the Reitan's test. It can be considered that brain function impairment is caused by prolonged alcohol ingestion or long-term viral damage, derived into cirrhosis and portal hypertension. ${ }^{20}$ These agents induce alterations in several brain structures and cellular pathways, causing mild or important cognitive defects.

The detailed psychometric or neuropsychological examination carried out in this study revealed important cognitive impairment in most of the 43 patients with liver disease.

The most outstanding findings were failures related to the constructional ability and copy capacity (recognition as identical or nonidentical designs). We found an important number of mistakes in recognition in the cube and scanning tests.

The use of this group of tests allowed documenting the existence of very poor performance in the visual motor area, corresponding to coordinated functions between visual motor and spatial areas. These findings are related to a serious deterioration in the velocity (speed) of mental processes during perception and discrimination of objects.

An alteration in the conjugated ocular movements during the act to follow an object can be suggested to have a diagnostic value during the presence of a subclinical hepatic encephalopathy.

The alteration in the similarity test indicates the presence of a marked defect to conform categories, suggesting an altered capacity of comprehension and a defect in the associative capacity in verbal concept that implies a deficiency in conceptual judgment.
FIGURE 4. Cubes

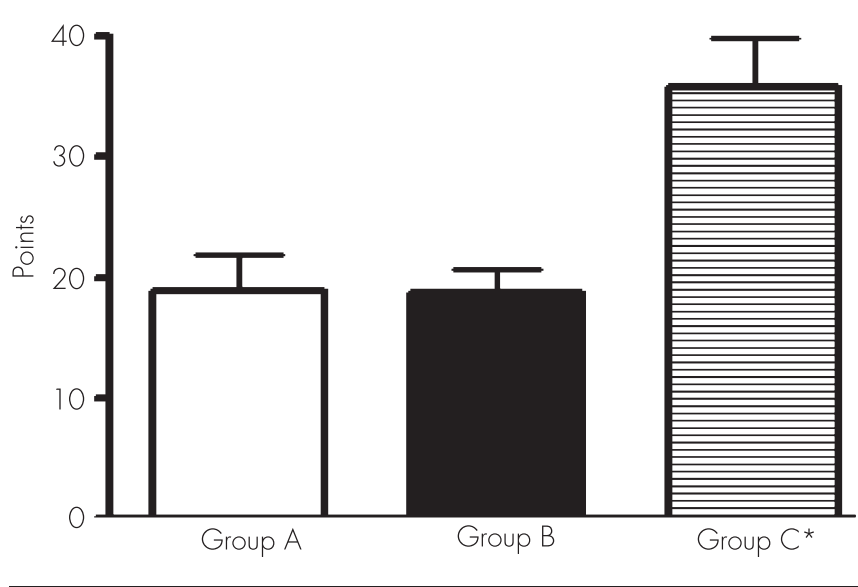

Chronic alcohol ingestion acts on numerous pathways that affect the function of the liver and other organs, including the brain. ${ }^{10}$ The main metabolite of alcohol, acetaldehyde, and several adducts, malonaldehyde and 4-hydroxy-2-nonenal, damage cell structure and organelles in the liver and brain, affecting microtubular proteins and inhibiting microtubule polymerization. It has been suggested that during this damage, excessive generation of reactive oxygen species overpasses the antioxidant system, forming the so-called oxidative stress. Reactive oxygen species alter membrane structure, lipid molecules, and DNA, producing membrane lipo-oxidation and affecting lipid metabolism.

Taken together, these findings suggest that alcohol intake induces oxidative stress and constitutes a pivotal factor in the development of liver and brain injury.

Viral damage, like hepatitis B and C viruses, probably uses a similar mechanism to produce intellectual deficits through its own toxins. The presence of hepatitis B or C virus in the patient may act on brain tissue by either the action of viral toxic substances or the presence of cytokines or chemokines released by macrophages or other cells, which are mainly the liver Kupffer cells. These two factors may act on brain nuclei, causing functional impairment. ${ }^{21}$ This speculation is based on the fact that infection induces neuroinflammation and brain dysfunction. Fatigue, mood disturbances, and cognitive dysfunction are frequent in patients infected with hepatitis $C$ virus who have mild liver disease. The pathological mechanism is still unclear. Viral sequences and proteins have been previously found in brain macrophage/microglia cells. Bokemeyer et al. ${ }^{21}$ 
found glial activation and macrophage infiltration in chronic inflammation and virus infections of the brain, endorsing the hypothesis that hepatitis $C$ virus infection may induce neuroinflammation. Wilkinson et al. ${ }^{22}$ also found evidence for activation of brain macrophages/microglia cells in autopsy brain tissue from hepatitis $C$ virus-positive patients.

Some of these mechanisms can be studied using animal models. However, the extrapolation of the findings, from animals to humans, remains a difficult task, because reactive oxygen species production and antioxidant status in humans is affected by severe nutritional environmental defects and drug influences, which are difficult to reproduce in animals.

Nevertheless, several investigations have found that the administration of antioxidant agents that reduce the levels of free iron or of agents that replenish glutathione levels can prevent or ameliorate the toxic effect of alcohol. The most convincing data, which indicate that oxidative stress contributes to alcohol liver disease, have come from studies using the intragastric alcohol infusion model. In these studies, alcohol liver disease was associated with enhanced lipid peroxidation, protein modification, formation of the 1-hydroxy-ethyl radical and lipid radicals, and a decrease in the hepatic antioxidant defense, particularly glutathione levels. ${ }^{23-25}$ Moreover, changes in the animal diet promote or reduce oxidative stress, leading to the corresponding changes in the extent of liver injury, as occurs when polyunsaturated fats, which are required for lipid peroxidation production, are replaced with saturated fatty acids ${ }^{26}$ and perhaps on brain damage also.

According to previous experiments from our laboratory, brain regions, specifically the hippocampus and prefrontal region, are involved in metabolic, functional, and morphological derangements in a portal hypertensive rat model, which reproduces this major cirrhotic complication using a simple stricture of the portal vein. ${ }^{27}$

The hippocampal region is a brain median temporal lobe formation that takes part in the processing of memory, behavior, and learning. It is also related to other parts of the brain, such as the brain cortex and amygdala. Thus, this brain region is probably involved in brain damage by different toxins and possibly participates in intellectual derangements.

Rusakov et al. ${ }^{28}$ studied the correlation of spatial learning in the rat hippocampus with synaptic ultrastructural appearance and suggested that it is possible to recognize brain areas and even cell populations in particular learning paradigms.

Stubley-Weatherly et al. ${ }^{29}$ examined the effects of hippocampal lesions using water maze performance in rats and found that both the dorsal CA1 and ventral CA3 subregions play important roles in learning.

Rats maintained in a spatially enriched environment show improved performance in a water maze task, which is associated with an increased density of dendritic spines in CA1 pyramidal cells, indicating an increased number of newly formed synapses associated with spatial learning. These results provide evidence that memory formation, at least in rats, may involve morphological and topographic changes in some neural circuits.

Unexpectedly, the memory function, analyzed for a short time or long time, shows minor alterations when comparing cirrhotic patients with control subjects, but visual memory was affected. ${ }^{4,5,30}$ Perhaps the lack of a significant failure in memory can be due to lesser damage to the hippocampus region. It would be interesting to study hippocampus behavior and its basic influence on memory in alcoholic and nonalcoholic chronic liver disease.

When we used the Boston Naming Test with our patients, we observed that it took a significantly long period of time for the patients to complete the answer, indicating the presence of a slower mechanism of thinking (patients must observe the object to remember its name). This kind of behavior is called paraphrasis and demonstrates the capacity of the patient to scan the information. Retrograde semantic memory is involved in this procedure. During depression, this test can show a normal result, but in dementia, scarce verbal fluency can be present.

The analysis of our results, after the application of this battery of tests, demonstrated that patients with chronic liver disease, from either alcoholic intake or viral damage, had an important impairment in their attention and mental concentration. Interestingly, mental alterations were documented when tests were applied to involve the praxis aspects (motor activity) and those that evaluate the velocity of the mental processes.

The psychoneurological symptoms that appear during chronic liver disease may correspond to the presence of several and complex mechanisms. Peterson et al. ${ }^{14}$ and Lozeva et al. ${ }^{31,32}$ reported that neurotransmitter systems, particularly alterations in the glutaminergic and monoaminergic receptors, are implicated, as shown in brains of cirrhotic patients and experimental models. ${ }^{33}$

The role of these pathways is to mediate important brain functions, such as the regulation of the sleep-wake cycle and the act of learning and memory.

Weissenborn et al. ${ }^{4}$ showed that, as the degree of cognitive impairment in cirrhotic patients without clinical 
signs of hepatic encephalopathy increases, the glucose use of the frontal and parieto-occipital cortex decreases. Because the brain metabolism of glucose is closely related to liver glycogen, the results of Weissenborn et al. are very interesting.

Cirrhotic liver and its scar and nodular formation decrease the number of normal hepatocytes and their function, independently of the etiology: alcohol or toxins from virus. On one hand, less glycogen is stored, with the possibility of sending less glucose to the brain; on the other hand, as occurs in long-term alcoholic consumption, there is a decrease in glutathione levels in the mitochondria.

The neuropsychological tests that are used in this research confirmed the presence of cognitive impairment in patients with liver cirrhosis due to alcoholic prolonged intake or hepatitis B or C virus damage. The presence of oxidative stress must be assumed in these patients as part of the mechanism of cognitive alterations. According to the literature, $51 \%-70 \%$ of cirrhotic patients present cognitive alterations. Animal models exposed to ammonium acetate show an important decrease in the activity of glutathione peroxidase in the liver and brain, which is the same as liver peroxidation. Ammonia is toxic to brain function and is the result of urea metabolism in the damaged liver that is caused by cirrhosis.

Corbalán et al. $^{34}$ found that the nitric oxide-guanylate cyclase signal transduction pathway is altered in brains of patients with chronic liver damage and that these alterations are different depending on the brain area involved.

An increase in $\mathrm{Ca}^{2+}$ intracellular concentration, which stimulates glutamate receptors ( $N$-methyl-D-aspartate), can also modify the signal transduction pathway in the brain. The entry of $\mathrm{Ca}^{2+}$ into CNS cells triggers the production of nitric oxide by neuronal nitric oxide synthase activation. This molecular alteration can produce different levels of intellectual changes, with coma being the most serious event in cirrhotic patients.

Injection of cyclic GMP, a hyperglycemic agent, causes an increase in brain glucose, ameliorating brain function.

According to the results by Erceg et al., ${ }^{35}$ the intracerebral injection of cyclic GMP to chronic hyperammonemic rats restores the ability to learn and memorize, using a Y-maze conditional discrimination task. The administration of an anti-inflammatory drug, such as ibuprofen, also contributes to the amelioration of learning, using the same Y-maze. ${ }^{36}$ We then suggest that both cyclic GMP and ibuprofen act on the impairment of the glutamate-nitric oxide-cyclic GMP pathway in the brain and, by different mechanisms, restore learning capacity.

\section{CONCLUSIONS}

A subtle decline in cognitive function may be an important cause of decreased psychological and working ability in cirrhotic patients. The alterations in cognitive function, as those found in our patients, can be subtle and not readily recognized but may initiate a subclinical encephalopathy.

We suggest that the cognitive function alterations found in our cirrhotic patients with either chronic alcohol intake or viral damage are associated with two different mechanisms: one related to alcohol or viral etiology and the other, and most important, to the cirrhotic liver, with alterations in fundamental pathways (glucose metabolism) that involve brain function.

\section{References}

1. Bernthal P, Hays A, Tarter RE, et al: Cerebral CT scan abnormalities in cholestatic and hepatocellular disease and their relationship to neuropsychologic test performance. Hepatology 1987; 7:107-114

2. McCrea M, Cordoba J, Vessey G, et al: Neuropsychological characterization and detection of subclinical hepatic encephalopathy. Arch Neurol 1996; 53:758-763

3. Quero JC, Hartmann IJ, Meulstee J, et al: The diagnosis of subclinical hepatic encephalopathy in patients with cirrhosis using neuropsychological tests and automated electroencephalogram analysis. Hepatology 1996; 24:556-560

4. Weissenborn K, Ennen JC, Schomerus H, et al: Neuropsychological characterization of hepatic encephalopathy. J Hepatol 2001; 34:768-773

5. Weissenborn K, Heidenreich S, Giewekemeyer K, et al: Memory function in early hepatic encephalopathy. J Hepatol 2003; 39:320-325

6. Amodio P, Quero JC, Del Piccolo F, et al: Diagnostic tools for the detection of subclinical hepatic encephalopathy: comparison of standard and computerized psychometric tests with spectral-EEG. Metab Brain Dis 1996; 11:315-327

7. Amodio P, Marchetti P, Del Piccolo F, et al: Visual attention in cirrhotic patients: a study on covert visual attention orienting. Hepatology 1998; 27:1517-1523

8. Amodio P, Del Piccolo F, Marchetti P, et al: Clinical features and survivial of cirrhotic patients with subclinical cognitive alterations detected by the number connection test and computerized psychometric tests. Hepatology 1999; 29:1662-1667

9. Fromenty B, Grimbert S, Mansouri A, et al: Hepatic mitochondrial DNA deletion in alcoholics: association with microvesicular steatosis. Gastroenterology 1995; 108:193-200

10. Wu D, Cederbaum AI: Alcohol, oxidative stress, and free radical damage. Alcohol Res Health 2003; 27:277-284 
11. Forton DM, Thomas HC, Murphy CA, et al: Hepatitis C and cognitive impairment in a cohort of patients with mild liver disease. Hepatology 2002; 35:433-439

12. Tarter RE, Hegedus AM, Van Thiel DH, et al: Nonalcoholic cirrhosis associated with neuropsychological dysfunction in the absence of overt evidence of hepatic encephalopathy. Gastroenterology 1984; 86:1421-1427

13. Pantiga C, Rodrigo LR, Cuesta $M$, et al: Cognitive deficits in patients with hepatic cirrhosis and in liver transplant recipients. J Neuropsychiatry Clin Neurosci 2003; 15:84-89

14. Peterson C, Giguere JF, Cotman CW, et al: Selective loss of $\mathrm{N}$-methyl-D-aspartate-sensitive L-[3H]glutamate binding sites in rat brain following portacaval anastomosis. J Neurochem 1990; 55:386-390

15. Pugh RNH, Murray-Lyon IM, Dawson JL, et al: Transection of the oesophagus for bleeding oesophageal varices. Br J Surg 1973; 60:646-649

16. Folstein MF, Folstein SE, McHugh PR: "Mini-mental state". A practical method for grading the cognitive state of patients for the clinician. J Psychiatr Res 1975; 12:189-198

17. Reitan RM: The relation of the trail making test to organic brain damage. J Consult Psychol 1955; 19:393-394

18. Reitan R: Trail Making Test: Manual for Administration and Scoring. Tucson, AZ, Reitan Neuropsychology Laboratory, 1979

19. Kaplan E, Goodglass H, Weintraub S: Boston Naming Test. Philadelphia, PA, Lea and Febiger, 1983

20. Eizayaga F, Scorticati C, Prestifilippo JP, et al: Altered bloodbrain barrier permeability in rats with prehepatic portal hypertension turns to normal when portal pressure is lowered. World J Gastroenterol 2006; 12:1367-1372

21. Bokemeyer M, Ding XQ, Goldbecker A, et al: Evidence for neuroinflammation and neuroprotection in HCV infectionassociated encephalopathy. Gut 2011; 60:370-377

22. Wilkinson J, Radkowski M, Eschbacher JM, et al: Activation of brain macrophages/microglia cells in hepatitis C infection. Gut 2010; 59:1394-1400

23. Tsukamoto H, Takei $\mathrm{Y}, \mathrm{McClain} \mathrm{CJ}$, et al: How is the liver primed or sensitized for alcoholic liver disease? Alcohol Clin Exp Res 2001; 25(Suppl ISBRA):171S-181S

24. Iimuro Y, Bradford BU, Yamashina S, et al: The glutathione precursor L-2-oxothiazolidine-4-carboxylic acid protects against liver injury due to chronic enteral ethanol exposure in the rat. Hepatology 2000; 31:391-398
25. Nanji AA, Zhao S, Lamb RG, et al: Changes in cytochromes P450, 2E1, 2B1, and 4A, and phospholipases $\mathrm{A}$ and $\mathrm{C}$ in the intragastric feeding rat model for alcoholic liver disease: relationship to dietary fats and pathologic liver injury. Alcohol Clin Exp Res 1994; 18:902-908

26. Feoli AM, Siqueira I, Almeida LM, et al: Brain glutathione content and glutamate uptake are reduced in rats exposed to pre- and postnatal protein malnutrition. J Nutr 2006; 136:2357-2361

27. Lores-Arnaiz S, Perazzo J, Prestifilippo J, et al: Hippocampal mitochondrial dysfunction with decreased mtNOS activity in prehepatic portal hypertensive rats. Neurochem Int 2005; 47:362-368

28. Rusakov DA, Davies HA, Harrison E, et al: Ultrastructural synaptic correlates of spatial learning in rat hippocampus. Neuroscience 1997; 80:69-77

29. Stubley-Weatherly L, Harding JW, Wright JW: Effects of discrete kainic acid-induced hippocampal lesions on spatial and contextual learning and memory in rats. Brain Res 1996; 716:29-38

30. Weissenborn K, Rückert N, Hecker $\mathrm{H}$, et al: The number connection tests $\mathrm{A}$ and $\mathrm{B}$ : interindividual variability and use for the assessment of early hepatic encephalopathy. J Hepatol 1998; 28:646-653

31. Lozeva V, Valjakka A, Lecklin A, et al: Effects of the histamine $\mathrm{H}(1)$ receptor blocker, pyrilamine, on spontaneous locomotor activity of rats with long-term portacaval anastomosis. Hepatology 2000; 31:336-344

32. Lozeva V, Tuomisto L, Sola D, et al: Increased density of brain histamine $\mathrm{H}(1)$ receptors in rats with portacaval anastomosis and in cirrhotic patients with chronic hepatic encephalopathy. Hepatology 2001; 33:1370-1376

33. Acosta GB, Fernández MA, Roselló DM, et al: Glutamine synthetase activity and glutamate uptake in hippocampus and frontal cortex in portal hypertensive rats. World J Gastroenterol 2009; 15:2893-2899

34. Corbalán R, Chatauret N, Behrends $S$, et al: Region selective alterations of soluble guanylate cyclase content and modulation in brain of cirrhotic patients. Hepatology 2002; 36:1155-1162

35. Erceg S, Monfort P, Hernandez-Viadel M, et al: Restoration of learning ability in hyperammonemic rats by increasing extracellular cGMP in brain. Brain Res 2005; 1036:115-121

36. Cauli O, Rodrigo R, Piedrafita $\mathrm{B}$, et al: Inflammation and hepatic encephalopathy: ibuprofen restores learning ability in rats with portacaval shunts. Hepatology 2007; 46:514-519 